\title{
Clinical databases and biobanks for etiologic and therapeutic studies in rheumatoid arthritis
}

\author{
Lars Klareskog
}

Let us assume that we want to prevent and treat rheumatic inflammatory diseases using the leads provided by experimental animal models, in which it is frequently possible to intervene very specifically. Let us also imagine the despair of the mouse experimentalist when we suggest that he or she randomly allocate treatments to animals of different genetic backgrounds and immunized with different disease triggers. This is close to how we currently treat complex, criteria-defined diseases, such as rheumatoid arthritis (RA). Although RA, for example, is defined by shared clinical features, these features can be caused by different molecular mechanisms, can encompass different immune reactions, and can be further complicated by comorbidities, such as cardiovascular diseases, infections or cancer.

I suggest that the construction of longitudinal clinical databases and accompanying biobanks is a necessary step towards making the techniques and therapies developed from mouse models available to human patients. Below follow three, admittedly biased, examples of how large databases and biobanks can contribute new insights when used in studies of etiologic factors, therapeutic effects, and outcomes.

In population-based cohorts of patients with arthritis, it has been demonstrated that an important environmental agent (smoking), in the context of a major susceptibility gene (the shared epitope of HLA-DRB1), might trigger immune reactions to citrulline-modified self proteins (Klareskog L et al. [2006] Arthritis Rheum 54: 38-46). Notably, the etiologic scenario involving these three contributing factors was seen in one major subpopulation of RA patients (defined by the presence of antibodies to cyclic citrullinated peptides), but not at all in other RA patients. The takehome message is that further immunologic studies aimed at understanding the molecular "....construction of longitudinal clinical databases and accompanying biobanks is a necessary step towards making the techniques and therapies developed from mouse models available

to human patients"

$L$ Klareskog is an Advisory Board member of Nature Clinical Practice Rheumatology.

Acknowledgments

The concepts in the present editorial have been discussed and partly begun to be implemented within an EU Biomed \& supported integrated project named AutoCure (www.autocure.org)

\section{Competing interests}

The author has declared he has no competing interests.

www.nature.com/clinicalpractice doi:10.1038/ncprheum0305 pathogenesis of RA should be performed in patients for whom we have appropriate data on genes, triggers and immunity-that is, patients included in good clinical databases and biobanks.

Efforts have been made to prevent the development of RA in patients with early undifferentiated arthritis by means of treatment with methotrexate: disease development was blocked, but only in the subset of patients who were seropositive for antibodies to anticyclic citrullinated peptides (van Dongen $\mathrm{H}$ et al. [2006] Ann Rheum Dis 65 (Suppl 2): 54). The take-home message is that subdividing groups of patients on the basis of etiological knowledge might be of value both in predicting response to therapy and in defining the appropriate patients to receive new generations of therapies directed towards pathway-specific targets.

Comparative databases containing clinical information from patients on traditional therapy versus biologics have demonstrated that treatment of RA with neutralizing agents against tumor necrosis factor is associated with a number of important complications, including infection, especially tuberculosis, and possibly lymphoma, as well as the mitigation of some comorbidities. This information is valuable in a clinical setting, but we still lack biological predictors for response to therapy and for adverse events. An urgent need, therefore, exists for large biobanks to be established in the context of the emerging clinical and epidemiologic surveillance systems for new drugs.

In summary, new initiatives, on both national and international levels, are needed to invest in prospective and, if possible, population-based databases and biobanks. Such initiatives will enable the collection of important information about rheumatic disease that can be used for studies of etiology, therapy and outcomes, and by basic scientists who are attempting to answer pertinent clinical questions more precisely. 